\title{
Anesthetic Management of a Patient With Situs Inversus for Bilateral Orthotopic Lung Transplantation
}

\author{
Emily G. Teeter, MD,* Brian P. Barrick, MD, DDS,* Priya A. Kumar, MD,* Benjamin E. Haithcock, MD, $†$ \\ Andrew R. Karenz, MD,* and Susan M. Martinelli, MD*
}

$\mathbf{P}$ RIMARY CILIARY DYSKINESIA (PCD), also known as immotile-cilia syndrome, is a rare, autosomal recessive disorder that affects several different organ systems. The underlying defect typically is a mutation in dynein, leading to abnormal ciliary structure and function. Patients with PCD may experience hearing loss, recurrent pulmonary infections, chronic sinusitis, and infertility because cilia are ubiquitous in the middle ear, respiratory tract, nasal passages, and reproductive tract. Cilia also are responsible for determining proper visceral laterality in utero. Thus, during embryogenesis in patients with PCD, visceral laterality will be determined at random, and half of these patients will have situs inversus totalis, or mirror-image visceral anatomy. ${ }^{1}$ An additional subset of patients with PCD will have situs ambiguous, wherein visceral anatomy is neither "typical" (situs solitus) nor mirrorimage (situs inversus). When present in combination with bronchiectasis and chronic sinusitis, situs inversus related to PCD represents Kartagener syndrome. ${ }^{2}$ The authors present the case of a patient with Kartagener syndrome who underwent bilateral orthotopic lung transplantation. Written informed consent to publish this case was obtained from the patient.

\section{CASE PRESENTATION}

The patient was a 53-year-old, 60-kg female with a known history of Kartagener syndrome. The manifestations of her PCD included the complete triad of Kartagener syndrome: bronchiectasis, sinusitis, and sinus inversus totalis. In addition, she had profound hearing loss. Before presentation, she was undergoing home supplemental oxygen therapy and had experienced chronic, recurrent lung infections with multidrugresistant Pseudomonas. Preoperatively, her forced expiratory volume in 1 second was $0.66 \mathrm{~L}$ (22\% of predicted) and her peak expiratory flow was $1.62 \mathrm{~L} / \mathrm{s}$ ( $25 \%$ of predicted). Her chest $\mathrm{x}$-ray was significant for dextrocardia and marked bronchiectatic changes (Fig 1). A preoperative chest computed tomography scan also was significant for bronchiectasis and mirror-image great vessel and pulmonary anatomy (Fig 2). Because of her worsening bronchioectasis secondary to PCD, the patient was evaluated and deemed appropriate to undergo double-lung transplantation.

When donor lungs became available, the patient was taken to the operating room, where American Society of Anesthesiologists standard monitors and a preinduction arterial line were placed. Notably, the electrocardiogram lead placement was reversed due to the patient's dextrocardia. General anesthesia was induced uneventfully, and a single-lumen endotracheal tube (ETT) initially was using a bronchoscope placed to facilitate adequate pulmonary clearance. This also provided clear visualization for placement of the double-lumen ETT. In addition to thick, purulent secretions, the bronchoscopy revealed mirror-image pulmonary anatomy. A proximal upper lobe bronchus and bronchus intermedius arose from the anatomic left lung, making it a "morphologic" right lung. In addition, the anatomic right lung had a long mainstem bronchus and was divided into upper and lower segments, making it a "morphologic" left lung (Video).

After bronchoscopy, the ETT was exchanged for a left-sided double-lumen ETT, which was advanced into the anatomic right (but morphologic left) mainstem bronchus with the tracheal lumen facing to the patient's left. Specifically, the ETT was advanced through the vocal cords, with the bronchial tip facing anteriorly, and was turned 90 degrees clockwise (rightward) as it was advanced. This enabled the bronchial lumen to pass into the anatomic right (morphologic left) lung with the tracheal lumen positioned appropriately above the carina. ETT position was confirmed using fiberoptic bronchoscopy. A transesophageal echocardiography (TEE) probe was placed without incident, and a left-sided internal jugular 9-Fr introducer catheter was placed using ultrasound guidance. TEE was used to confirm the presence of the wire in the superior vena cava before dilation and line placement. A pulmonary artery catheter was positioned easily, and proper position was confirmed using waveform analysis and TEE.

The TEE examination was complicated by the patient's dextrocardia and mirror-image anatomy, such that the midesophageal 4-chamber view, midesophageal ascending aorta short-axis view, and transgastric short-axis-views were obtained at 180 degrees in order for them to be displayed in their traditional orientation and lessen confusion when reviewing the TEE findings. Alternatively, the left-right screen invert could have been used for these 0-degree images. The remainder of the TEE views were obtained at an angle of $180^{\circ}$ minus typical omniplane angle.

Because the patient was at risk for additional congenital cardiac anomalies, a complete TEE examination was performed to rule out associated congenital findings such as persistent leftsided superior vena cava, anomalous pulmonary venous return, septal defects, and valvular abnormalities. In this case, the TEE examination was unremarkable except for moderate left ventricular hypertrophy, moderate tricuspid regurgitation, and moderate right ventricular dilation. Once the clamshell incision was made, ETT position was further verified using direct inspection of differential lung inflation in the surgical field. The surgical course was relatively uncomplicated, and the donor lungs were implanted in their native position (morphologic right lung in the right chest, followed by morphologic left lung

From the *Department Anesthesiology, and the tDepartment of Cardiothoracic Surgery, University of North Carolina at Chapel Hill, Chapel Hill, $N C$.

Address reprint requests to Emily G. Teeter, MD, University of North Carolina at Chapel Hill, N2198 UNC Hospitals CB 7010, Chapel Hill, NC 27599-7010. E-mail: eteeter@aims.unc.edu

(C) 2016 Elsevier Inc. All rights reserved. 1053-0770/2602-0033\$36.00/0

http://dx.doi.org/10.1053/j.jvca.2016.01.019

Key Words: Situs inversus, Kartagener syndrome, lung transplantation, dextrocardia 


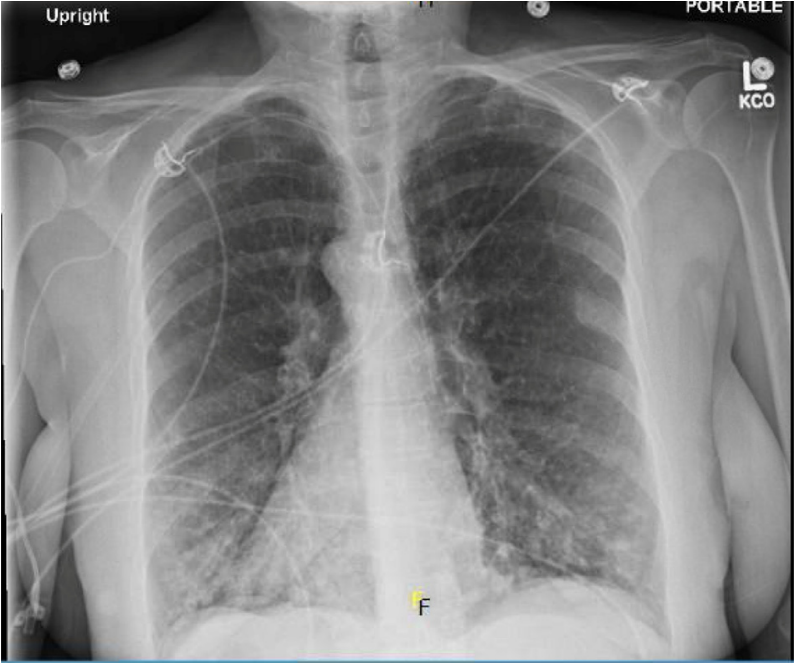

Fig 1. Preoperative chest $x$-ray showing hyperinflated lungs, flattening of bilateral hemidiaphragms, right-sided gastric bubble, and bronchiectasis.

in the left chest). At the conclusion of the procedure, the double-lumen ETT was exchanged for a single-lumen ETT and the patient was transported to the intensive care unit. The patient was extubated on postoperative day 1 , and a thoracic epidural catheter was placed with some difficulty for analgesia. However, the epidural did not provide adequate pain relief and was removed. The patient refused replacement, and her pain was well-controlled with hydromorphone patient-controlled analgesia. Despite a lengthy hospital course due to pulmonary infection, she was discharged home 17 days later. She was readmitted 1 week later for an episode of acute rejection, which was treated and resolved with high-dose steroids. The patient currently is at home and doing well.

\section{DISCUSSION}

Primary ciliary dyskinesia, and specifically Kartagener syndrome, is associated with a mutation in the protein dynein, found in the ciliary arms of many structures. During early embryologic development, the coordinated beating of cilia around the ventral node is responsible for a leftward flow of extraembryonic fluid. This flow triggers a rise in intracellular calcium in cells on the left side of the node, leading to asymmetric gene expression and morphogenesis. This is how the normal asymmetry of the viscera is established. ${ }^{3}$ Impaired ciliary function may result in situs inversus and other previously described problems.

Patients who have Kartagener syndrome are more likely to present for thoracic procedures as a result of the impaired function of their respiratory cilia. Like cystic fibrosis (CF), PCD is associated with recurrent, chronic pulmonary infections. CF patients typically experience bronchiectasis in the upper lobes, whereas in PCD, the lower lobes are more frequently affected. Unlike CF, PCD generally is associated with a normal life expectancy. However, as in this case, patients with PCD may encounter morbidity related to their lung disease and may require lung transplantation. ${ }^{1}$
From an anesthetic standpoint, patients with Kartagener syndrome pose several challenges. The associated dextrocardia in patients with situs inversus requires that electrocardiogram lead placement be reversed for proper analysis, such that left-sided limb leads and fifth precordial leads are placed on the right, and right-sided limb leads on the left. ${ }^{4}$ As for central venous access and pulmonary artery catheter placement, a left internal jugular vein approach may be more prudent. As opposed to patients with situs solitus, the left internal jugular vein provides the most direct access to the morphologic right atrium. Also, this avoids potential damage to the brachiocephalic vein, thoracic duct (both rightsided structures in this case), and right mammary vein.

Airway management may be complicated for 2 main reasons. First, as in $\mathrm{CF}$, these patients are prone to thick secretions as a result of the underlying ciliary dysfunction and bronchiectasis. Aggressive preoperative optimization and treatment of infection cannot be overemphasized. Therapeutic bronchoscopy through a large single-lumen ETT before double-lumen ETT placement is helpful. Second, mirror-image anatomy of the bronchi may make lung isolation more challenging. Bronchoscopic inspection of the bronchial anatomy through a single-lumen ETT is advantageous before a decision is made regarding lung isolation. ${ }^{6}$ In this patient, the bronchial lumen of a left-sided double-lumen ETT was placed into the anatomic right lung because bronchoscopy revealed that this was the morphologic left lung. The ETT functioned appropriately for the case. There have been additional case reports of both right- and left-sided double-lumen ETTs being used in this way, as well as alternative solutions to onelung ventilation, including bronchial blockers and modified single-lumen ETT placement. ${ }^{5,7}$

In the event of bilateral sequential lung transplantation, mirror-image anatomy may influence the surgeon's decision as to which lung to replace first. Usually the native lung with

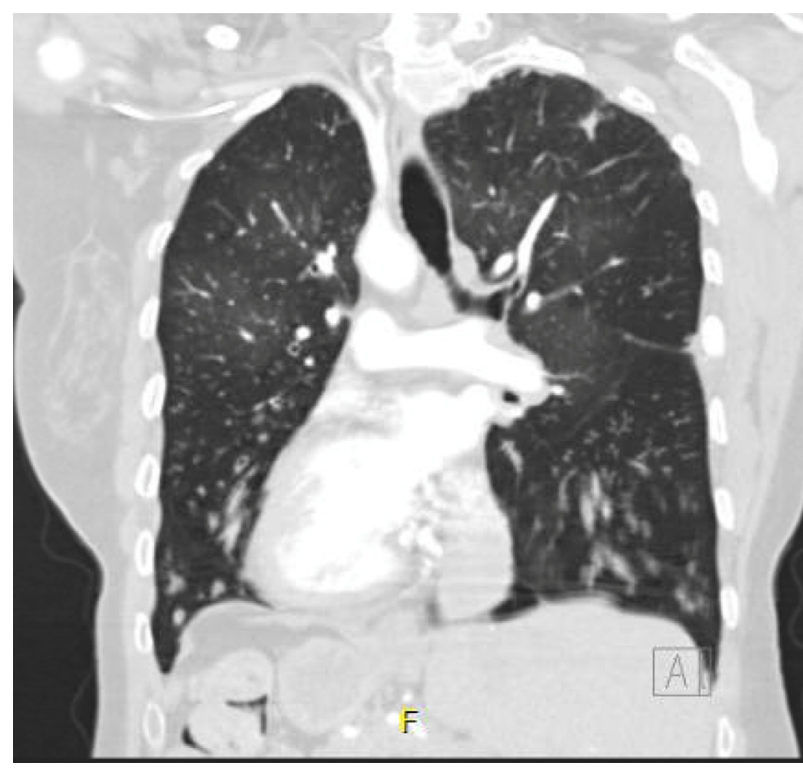

Fig 2. Coronal computed tomography scan of the chest illustrating takeoff of the right upper lobe from the left-sided lung. Also evident are severe bronchiectasis, dextrocardia, and right-sided aortic arch. 
worse function and/or perfusion is replaced first. In the setting of a relatively even perfusion split (as in this case) and situs inversus, the anatomic left lung likely would be replaced first due to the patient's dextrocardia. With dextrocardia, replacement of the left lung would be easier because the heart would not obstruct access. It should be kept in mind that, as with other patients with end-stage lung disease, lung transplant candidates with PCD have limited pulmonary reserve and may desaturate quickly, so airway management should be performed as expeditiously as possible.

As previously mentioned, TEE examination in situs inversus may be challenging and requires vigilance when differentiating morphologic and anatomic left/right structures, including the atria, ventricles, and pulmonary veins. Views that are obtained near 0 degrees, such as the midesophageal 4 chamber, midesophageal ascending aorta short axis, and transgastric short axis, will be mirror images of their textbook counterparts (Figs 3 and 4). To lessen confusion, the operator may choose to obtain these views at 180 degrees or invert left/right on the screen on the TEE machine so that they appear in their typical orientation. As previously mentioned, other "standard" views are obtained at an omniplane angle of $180^{\circ}$ minus typical omniplane angle. For example, the right ventricular inflowoutflow view, used to monitor right ventricular function and normally obtained at 60 to 75 degrees, is obtained at 105 to 120 degrees. The aortic valve short-axis view, which can be used to look for air at the coronary ostia during reperfusion and normally is obtained at 40 degrees, is best obtained at 140 degrees. The bicaval view, normally found at 110 degrees, is best obtained at 70 degrees while rotating the probe to the left because the morphologic right atrium is located to the left. ${ }^{8}$

During bilateral orthotopic lung transplantation, identification of the pulmonary veins is of particular importance. The anatomic right (but morphologic left) upper pulmonary vein will be found at approximately 90 to 120 degrees with a rightward turn of the probe. The ligament of Marshall ("Coumadin ridge") and left atrial appendage also may be visualized in this view (Fig 5A). The anatomic left (but

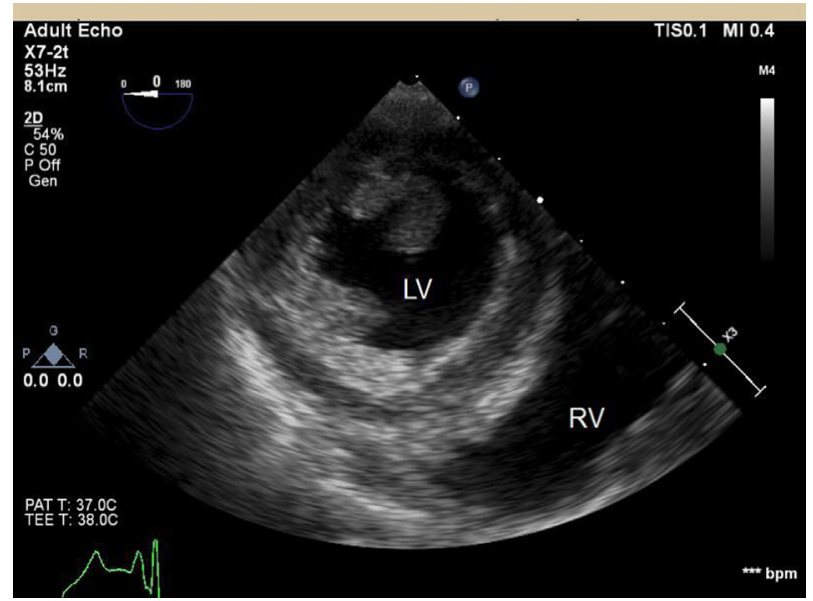

Fig 3. Transesophageal echocardiographic transgastric midpapillary view. The positions of the left ventricle (LV) and right ventricle (RV) are inverted, and the right ventricle appears dilated and hypertrophied.

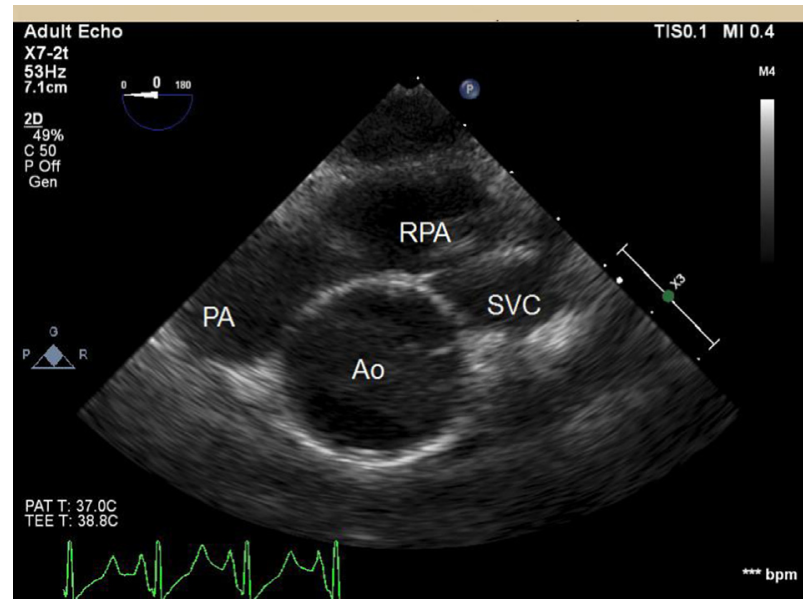

Fig 4. Transesophageal echocardiography midesophageal ascending aorta short-axis view. This view, found at 0 degrees, is the mirror image of the "typical" ascending aorta short-axis view, in which the superior vena cava (SVC) and right pulmonary artery (RPA) are found to the left of the screen. PA, main pulmonary artery; Ao, ascending aorta.

morphologic right) upper pulmonary vein, which typically is visualized from the bicaval view, should be found at approximately 70 to 80 degrees and with a leftward turn of the probe (Fig 5B). All 4 pulmonary veins should be located to the best of the operator's ability preoperatively to rule out anomalous venous return. Maximum systolic velocity should be measured postoperatively in each pulmonary vein to rule out anastomotic stenosis (maximum acceptable velocity: $1 \mathrm{~m} / \mathrm{s}$ ).

Also of importance is the ability to rule out other associated congenital cardiac anomalies, such as septal defects, valvular abnormalities, and transposition of the great arteries. In patients with Kartagener syndrome, congenital heart defects are present in $5 \%$ to $10 \%$ of those with dextrocardia and in more than $90 \%$ of patients with levocardia. ${ }^{8}$ PCD patients with severe lung disease may have longstanding pulmonary hypertension. Right ventricular dysfunction should be addressed before transplantation, and biventricular function regularly should be evaluated intraoperatively using TEE.

Thorough communication and explicit handoff among the surgical, anesthesia, operating room, and intensive care unit teams are imperative to ensure the continued safety of a patient with such abnormal anatomy. A thorough understanding of situs inversus is necessary when performing invasive procedures, defibrillation, and airway management in the perioperative setting, and these details often can be missed in handoff in the setting of such a complicated surgical procedure. $^{4}$

Finally, postoperative challenges in this subset of patients are common to all patients who have undergone lung transplantation. Respiratory insufficiency, pain control, rejection, infection, and pleural complications such as air leak and empyema are major contributors to morbidity and mortality. Similar to patients with CF, patients with PCD struggle with chronic infection, and this may be an issue extending from the preoperative to the postoperative period, as with this patient. 

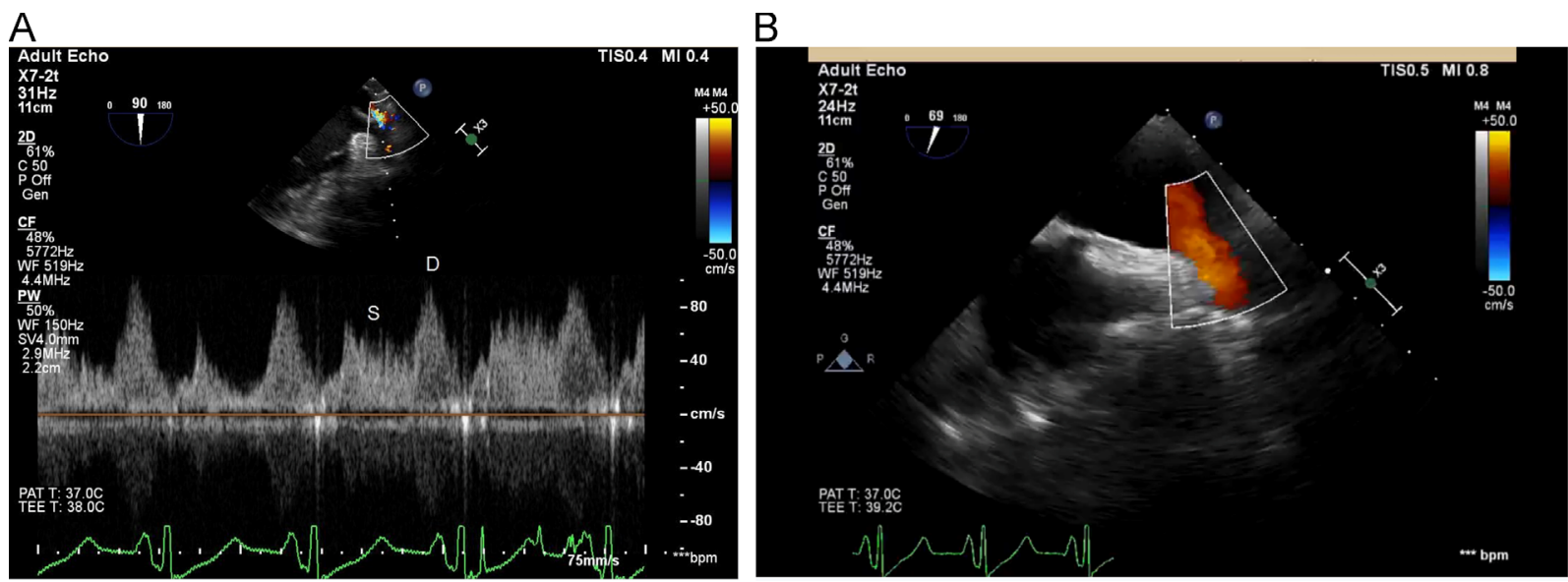

Fig 5. (A) Before transplant, the morphologic left (but anatomic right) upper pulmonary vein was found at the midesophageal position at approximately 90 degrees and by turning the probe to the right. The ligament of Marshall ("Coumadin ridge") and left atrial appendage also are visible in the inset. Here, the pulsed-wave Doppler cursor has been placed just within the pulmonary vein and reveals a pulmonary venous pattern with systolic blunting, likely due to diastolic dysfunction because no systolic dysfunction or significant mitral regurgitation was present. (B) Before transplant, the morphologic left (but anatomic right) upper pulmonary vein was found at the midesophageal position at approximately $\mathbf{7 0}$ degrees and by turning the probe to the left. S, systolic wave; $D$, diastolic wave.

\section{CONCLUSION}

In summary, patients with Kartagener syndrome present challenges to the anesthesia team related to monitoring, procedures, and airway management due to the associated primary ciliary dyskinesia and situs inversus. However, with careful planning and heightened vigilance, these patients can be anesthetized safely for even the most complicated surgical procedure, such as bilateral orthotopic lung transplantation.

\section{APPENDIX A. SUPPLEMENTARY INFORMATION}

Supplementary data associated with this article can be found in the online version at http://dx.doi.org/10.1053/j.jvca.2016. 01.019

\section{REFERENCES}

1. Lobo J, Zariwala M, Noone P: Primary ciliary dyskinesia. Semin Respir Crit Care Med 36:169-179, 2015

2. Skeik N, Jabr F: Kartagener syndrome. Int J Gen Med 12:41-43, 2011

3. Basu B, Brueckner M: Cilia multifunctional organelles at the center of vertebrate left-right asymmetry. Curr Top Dev Biol 85:151-174, 2008

4. Bajwa SJS, Kulshrestha A, Kaur J, et al: The challenging aspects and successful anaesthetic management in a case of situs inversus totalis. Indian J Anaesth 56:295-297, 2012

5. Tunç M, Sazak HG, Cengiz Z, et al: Thoracic anesthetic management of a patient with Kartagener's syndrome. Turk J Anaesth Reanim 40:290-293, 2012
6. Eapen S, Kar S, Kiran S, et al: Is fibreoptic bronchoscopy a must prior to one lung ventilation in a situs inversus patient? J Anaesthesiol Clin Pharmacol 29:404-405, 2013

7. Bougaki M, Orii R, Yamada Y: Right bronchial intubation using a left-sided double-lumen tube in a patient with situs inversus. Masui 56: 1411-1413, 2007

8. Raut MS, Maheshwari A, Shad S, et al: How standard transesophageal echocardiography views change with dextrocardia. Ann Card Anaesth 16:218-220, 2013 